Research articles

\title{
Non-uniform magnetization profile in ferromagnetic heterostructures leading to topological Hall effect like signatures
}

\author{
Nandana Bhattacharya, S. Middey* \\ Department of Physics, Indian Institute of Science, Bengaluru 560012, India
}

\section{A R T I C L E I N F O}

\section{Keywords:}

Magnetic hysteresis

Anomalous Hall effect

Topological Hall effect

Interfacial effects

\begin{abstract}
A B S T R A C T
Anomalous Hall effect (AHE), which arises when a current is passed through a ferromagnetic material subjected to a perpendicular magnetic field, is proportional to the magnetization of the sample. Additional hump-like features in AHE are often attributed to the presence of non-trivial spin textures leading to topological Hall effect (THE). However, several recent reports have emphasized in context of ferromagnetic $\mathrm{SrRuO}_{3}$ based heterostructures that the sample inhomogeneity can also result in THE-like features. In order to investigate this issue in general for any ferromagnetic heterostructure, we have considered a phenomenological model to calculate the changes in the shape of hysteresis loop due to various interfacial effects. These changes in the magnetization have been accounted for by considering that the interdomain magnetic coupling parameter ( $\alpha$ ) varies exponentially with the distance from the interface along the growth direction of the heterostructure. In case of symmetric interfaces on both sides of a ferromagnet, we have considered the variation of $\alpha$ as a Gaussian function. We have found that the additional AHE contribution due to the net change in magnetization in such cases are akin to experimentally observed THE, even though we have not considered any topological quantity explicitly in our model. Thus, we propose another situation with nonuniform magnetization profile that may be used to explain additional features in AHE, which might not necessarily be intrinsic THE.
\end{abstract}

\section{Introduction}

Transition metal oxides (TMOs) are host of a plethora of collective phenomena such as magnetism, ferroelectricity, metal-insulator transition, unconventional superconductivity, charge ordering, etc [1-4]. The tremendous advancement in thin film growth technologies over the last two decades have enabled the research community to grow artificial structures of these complex oxides with unit cell precision [5-11]. Such heterostructuring [9,12-25] leads to subtle modifications in spin, charge, orbital, and lattice sectors at the interface, resulting in a variety of emergent magnetic behaviors such as interfacial ferromagnetism, exchange bias, spin spiral magnetic phase, enhanced magnetic ordering temperature, topological Hall effect (THE), etc. THE arises in materials with non-zero scalar spin chirality $\left[\chi_{i, j, k}=S_{i} \cdot\left(S_{j} \times S_{k}\right), S_{i}\right.$ denotes localized moment], which results in an internal fictitious magnetic field due to the real space Berry phase. Since the first report of the observation of THE in MnSi [26], THE has been demonstrated in a variety of systems having chiral spin configurations [27-31]. As the simultaneous presence of broken inversion symmetry and finite spin orbit coupling (either intrinsic or Rashba-type or both) can lead to a sizeable Dzyaloshinskii-Moriya (D-M) interaction, interfacial engineering of TMOs has become a successful approach to achieve THE [25,32-34].
Bulk $\mathrm{SrRuO}_{3}$ (SRO) is a metallic ferromagnet with a magnetic transition temperature of $165 \mathrm{~K}$ [35]. The origin of THE-like signal in SRO-based heterostructures is under intense scrutiny in recent years [25,36-45]. While the origin of this phenomenon has been linked with the stabilization of nontrivial spin textures like skyrmions near the interface [25,36-39], another set of studies have emphasized that the sample inhomogeneity gives rise to THE-like features [40-44]. Since the shape of ferromagnetic hysteresis loop is generally affected due to the electronic, magnetic and structural changes across the interface $[46,47]$, we have considered another possible scenario with a non-uniform magnetization profile along the growth direction of the heterostructure [48]. We have examined whether layer dependent hysteresis loops in such systems can lead to these THE-like signature without invoking any topological interpretation.

In this work, we have simulated the hysteresis loops using a phenomenological approach for a ferromagnet having layer dependent internal molecular field (we refer one unit cell along the growth direction as 'layer' throughout the paper). We have followed the mathematical model of Jiles et al. (Ref. [49]), which was developed to calculate the hysteresis loop for an isotropic ferromagnet. In the simplest form, the effective magnetic field felt by an individual domain within a

\footnotetext{
* Corresponding author.

E-mail address: smiddey@iisc.ac.in (S. Middey).
} 
ferromagnet can be expressed as $\boldsymbol{H}_{\boldsymbol{e}}=\boldsymbol{H}+\alpha \boldsymbol{M}$, where $\boldsymbol{H}$ is the actual magnetic field within the domain and $\boldsymbol{M}$ represents the total sample magnetization per unit volume. The interdomain coupling is represented by a mean field parameter $\alpha$. It is assumed here that the change in the magnetization due to any interfacial effect can be accounted phenomenologically by considering $\alpha$ to be non-uniform and strongly dependent on the distance $(z)$ from the interface. For a ferromagnet, having an interface on one side, hysteresis curves have been simulated considering that $\alpha$ varies exponentially with $z$ below a critical length scale $z_{0}$ and it becomes equal to $\alpha_{0}$ (mean field parameter of an uniformly magnetized ferromagnet) above that. To investigate effect of symmetric interfaces on both sides of a ferromagnet, $\alpha$ has been considered to vary as a Gaussian function. We have found that the additional AHE, which is proportional to the change in magnetization $\Delta M$ (defined as the change in magnitude of magnetization between the cases with constant and layer-dependent $\alpha$ ), appears very similar to what have been claimed experimentally as THE signature in several systems. [33,39,50-53].

\section{Details of simulation}

Under the assumption of the presence of non-trivial spin textures like skyrmions [25,36-39], the Hall resistivity $\left(\rho_{H}\right)$ is expressed as

$\rho_{H}=\rho_{\mathrm{OHE}}+\rho_{\text {additional }}=\rho_{0} H+\rho_{\text {additional }}$

where $\rho_{\text {additional }}=\rho_{\mathrm{AHE}}+\rho_{\mathrm{THE}}=R_{a} \boldsymbol{M}+\rho_{\mathrm{THE}}$ with $\rho_{\mathrm{OHE}}, \rho_{\mathrm{AHE}}, \rho_{\mathrm{THE}}$ denoting ordinary Hall effect (OHE), AHE and THE, respectively. $\rho_{0}, R_{a}$ are constant and $M$ is the magnetization.

In case of a heterostructure with nonuniform magnetization along the growth direction, the total magnetization $(M)$ can be considered as summation of two terms: $M^{\prime}$ (magnetization of a uniform ferromagnet) and $\Delta M$ (this corresponds to the change in $M^{\prime}$ due to all interfacial effects). In such system, we can also express $\rho_{\text {additional }}$ as a combination of two AHE contributions instead of considering any intrinsic THE

$\rho_{\text {additional }}=R_{a} M^{\prime}+R_{a} \Delta M$

We have simulated hysteresis loops for $M$ and $M^{\prime}$ using the method described in Ref. [49], which we briefly discuss here. The magnetization $\boldsymbol{M}$ is related to the $\boldsymbol{H}_{\boldsymbol{e}}$ and can be written as

$\boldsymbol{M}=\boldsymbol{M}_{s} f\left(H_{e}\right)$

where $f\left(H_{e}\right)$ is an arbitrary function with the following properties: $f \rightarrow 0$ when $H_{e}=0$ and $f \rightarrow 1$ as $H_{e} \rightarrow \infty$. $\boldsymbol{M}_{s}$ is the saturation magnetization. Eq. (3) does not consider any hindrance to the change in magnetization such as pinning of domain wall motion; therefore, represents only the anhysteretic magnetization curve $\left(\boldsymbol{M}_{\boldsymbol{a n}}\right)$ of a ferromagnet in reality.

Thus, one may rewrite Eq. (3) as

$\boldsymbol{M}_{a n}\left(H_{e}\right)=\boldsymbol{M}_{s} f\left(H_{e}\right)$

Jiles et al. [49] further modeled $\boldsymbol{M}_{a n}$ by a modified Langevin function [54]

$\boldsymbol{M}_{a n}\left(H_{e}\right)=\boldsymbol{M}_{s}\left[\operatorname{coth}\left(H_{e} / a\right)-\left(a / H_{e}\right)\right]$

The shape of the anhysteretic magnetization is determined by the parameter $a$, which has the same dimension as $H_{e}$.

In presence of an external magnetic field, the size of ferromagnetic domains, which are oriented along the direction of the field, would grow in size. However, pinning sites such as structural imperfections, impurity atoms, etc. disrupt domain wall motion and result in irreversible changes in magnetization curves. The energy loss per unit volume due to the pinning can be represented as (see Ref. [49] for the derivation)

$E_{p i n}(M)=K \int_{0}^{M} d M^{\prime}$
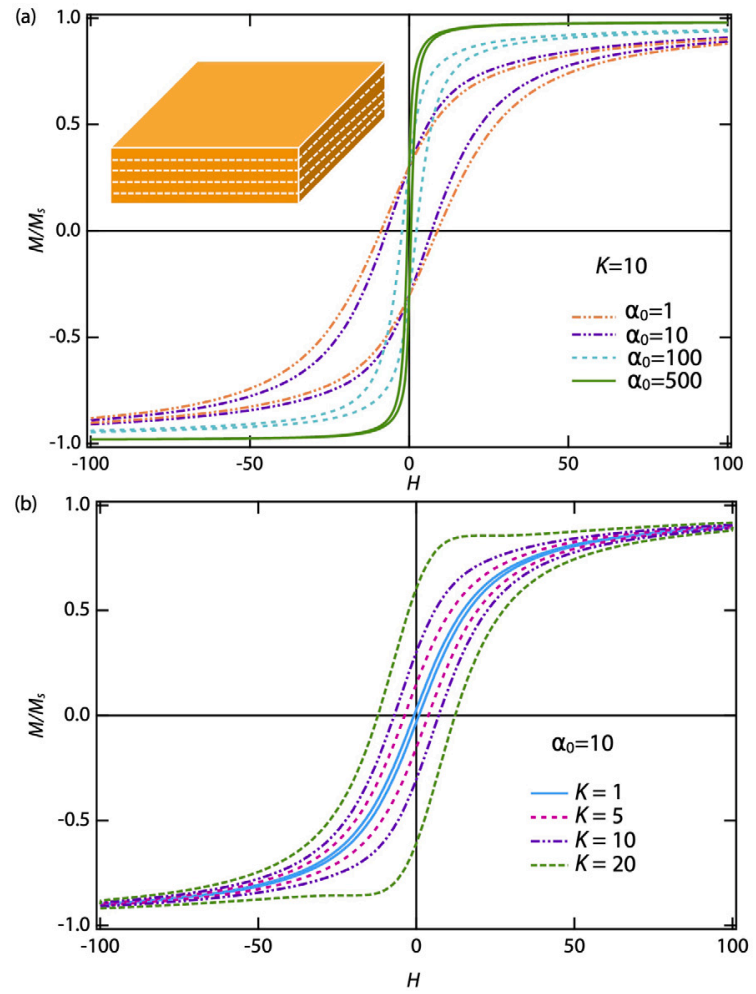

Fig. 1. Simulated hysteresis curves of a uniform ferromagnet (without any interface): (a) for different values of $\alpha_{0}$ keeping $K=10$, (b) for different values of $K$ with $\alpha_{0}=10$.

where $K$ is a constant and related to the average density of pinning sites, the average pinning energy of all sites for $180^{\circ}$ oriented walls and the magnetization density of a domain aligned along the direction of the field.

The magnetization energy is defined as the difference between the ideal, lossless case $\int M_{a n}\left(H_{e}\right) d B_{e}\left(B_{e}\right.$ is the effective magnetic induction) and the loss due to hysteresis $K \int d M$ i.e.

$\int M d B_{e}=\int M_{a n}\left(H_{e}\right) d B_{e}-K \int \frac{d M}{d B_{e}} d B_{e}$

The corresponding differential form of the equation is

$M=M_{a n}-\beta K \frac{d M}{d B_{e}}$

where $\beta$ is $+1(-1)$ for the positive (negative) sweep of $H$ as the pinning process opposes the direction of the applied field. This is the master equation that we have used to simulate the hysteresis curve. Since both sides of Eq. (8) contain $M$, we have evaluated first a trial magnetization $M_{1}$ as a function of external magnetic field $H$ by the relation $M_{1}(H)=M_{s}[\operatorname{coth}(H / a)-(a / H)]$. The effective field is treated as $H_{e}=H+\alpha M_{1}$. We have used Eqs. (5) and (8) sequentially to simulate hysteresis loops. Also, we have considered $H_{e}$ in Gaussian units, hence $B_{e}=H_{e}$ and so $\frac{d M}{d B_{e}}$ can be written as $\frac{d M}{d H_{e}}$. The derivative of $M$ with respect to $H_{e}$ has been calculated using the method of finite differences [55]. The parameter $a$ is taken as 11 through out the paper.

\section{Results and discussions}

We first discuss the results of hysteresis loop simulation for a ferromagnet without any interfacial effects [see inset of Fig. 1(a)]. For this, the material has been considered to be consisting of $N=100$ unit cells along the growth direction. The total magnetization has been evaluated by summing the magnetization of each individual unit cell, characterized by a same constant value of the mean field parameter 

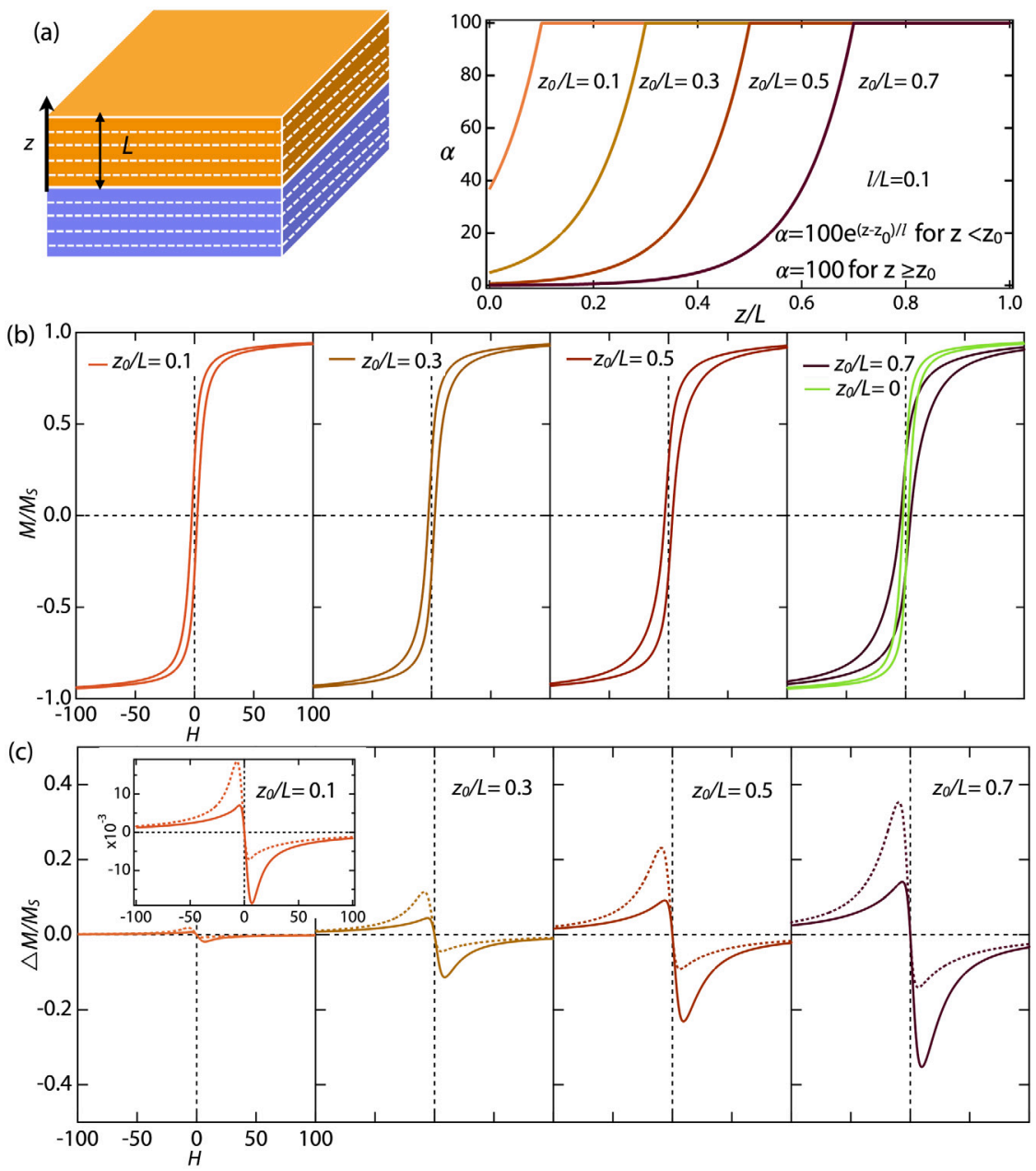

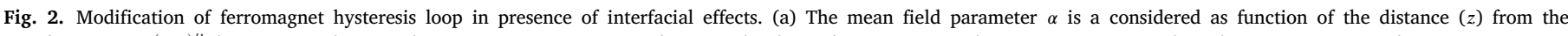

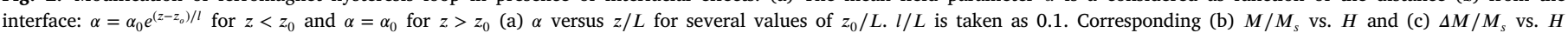

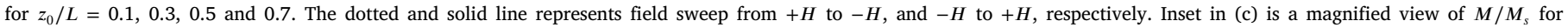
$z_{0} / L=0.1$.

$\alpha=\alpha_{0}$. Fig. 1(a) summarizes the results of simulation for different values of $\alpha_{0}$ for a fixed value of the parameter $K=10$. While the remanent magnetization $\left(M_{R}\right)$ is independent of $\alpha_{0}$, the coercive field $\left(H_{c}\right)$ decreases with the increase in $\alpha_{0}$. Moreover, a lower value of $\alpha_{0}$ generates the typical 'sigmoid' type ferromagnetic hysteresis loop, whereas the magnetization approaches to its saturation value very near to the zero field when larger values of $\alpha_{0}$ (e.g. 500) are considered in our simulation. Thus, the parameter $\alpha_{0}$ can be tuned to study soft and hard ferromagnets, having same $M_{R}$. Since the parameter $K$ represents the energy loss due to all pinning processes, our simulations for a fixed $\alpha_{0}$ found a decrease in both $H_{c}$ and $M_{R}$ with a lowering of the parameter $K$ [ Fig. 1(b)]. We have also simulated hysteresis with a larger range of $H$ for much larger values of $K$ and the results have been shown in the Supplementary Materials.

\subsection{Effect of interface on one side of the ferromagnet}

When the ferromagnetic material is grown on a substrate or heterostructured with another compound, the interfacial effects are very likely to affect the ferromagnetic response of the layers near the interface. We take into account such interfacial effects on the ferromagnetic hysteresis by considering that the parameter $\alpha$ is no longer uniform through out all unit cells along the $z$ directions. Since interfacial effects very often vary exponentially with the distance from the interface [13, $56,57]$, it is reasonable to assume that the $\alpha$ has the following exponential dependence with the distance from the interface $\alpha=\alpha_{0} e^{\left(z-z_{o}\right) / l}$ for $z<z_{0}$ and $\alpha=\alpha_{0}$ for $z>z_{0}$, where $z_{0}$ is a critical distance from the interface, above which interfacial effects are insignificant. $l$ is a parameter with the dimension of length. The corresponding variation of $\alpha$ across the ferromagnetic material is plotted in the right panel of Fig. 2(a) as a function of the fractional distance from the interface for different values of $z_{0} / L\left(l / L=0.1\right.$ and $\alpha_{0}=100$ were used for these plots).

The hysteresis curves for these distance dependent $\alpha$ with $K=10$ have been simulated for a ferromagnetic system, consisting of $N=100$ unit cells, similar to the previously discussed case of a uniform ferromagnet (Fig. 1). As expected, the hysteresis loop for the $z$-dependent $\alpha$ looks quite different compared to the case of the uniform ferromagnet [see the right most panel of Fig. 2(b)]. As we reduce the extent of interfacial effects by decreasing $z_{0} / L$, the hysteresis loop gradually becomes similar to the hysteresis of a uniform ferromagnet. This is further evident in Fig. 2(c), where the difference $(\Delta M)$ between the magnetization response of the layer dependent case and the layer independent case have been shown for the same set of $z_{0} / L$. Though the $\Delta M / M_{s}$ decreases with the reduction of $z_{0} / L$, the interfacial effect is still prominent even in case of the simulation with $z_{0} / L=0.1$ [see inset in Fig. 2(c)]. The hysteresis in $\Delta M / M_{s}$ (difference between forward and backward field sweep) also increases with $z_{0} / L$. Our most surprising finding is that these $\Delta M$ would lead to an extra contribution (= $R_{a} \Delta M$ in Eq. (2)) in the Hall effect measurement, which would resemble THE-features, reported in a variety of quantum materials in 

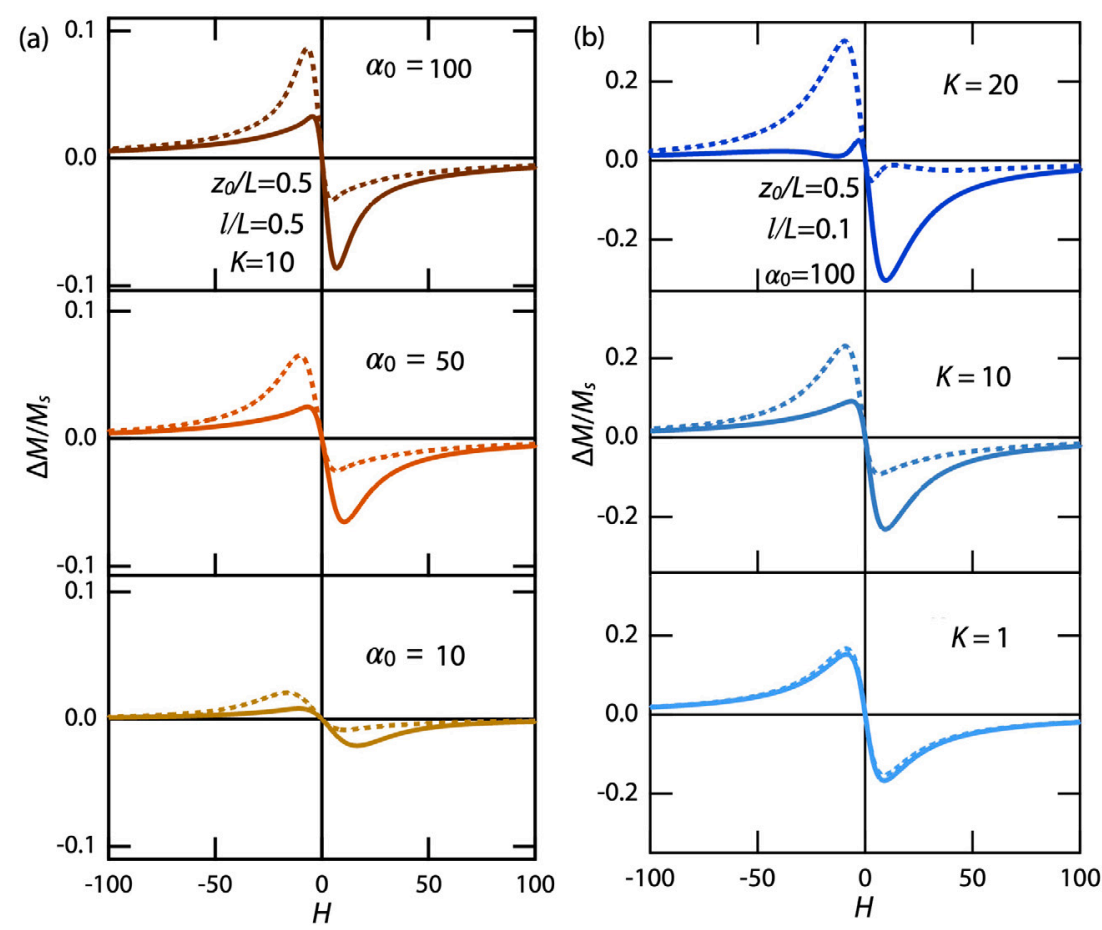

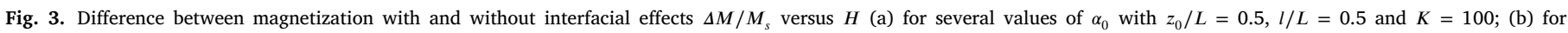
several values of $K$ with $z_{0} / L=0.5, l / L=0.1$ and $\alpha_{0}=100$. The dotted and solid line represents field sweep from $+H$ to $-H$, and $-H$ to $+H$, respectively.

recent years [33,39,45,50-53]. Interestingly, the $\Delta M$, obtained from our simulations, also varies linearly near $H=0$ - akin to the linear variation of $\rho_{T H E}$ observed experimentally [58].

The effect of the parameter $l$ has been explored with a fixed value of $z_{0} / L=0.5$ (results are shown in Supplementary Materials). As expected, smaller value of $l$ would correspond to a stronger interfacial effect, leading to an increase of $\Delta M / M_{s}$.

We have already demonstrated in context of a uniform ferromagnet (Fig. 1(a)) that we can explore both soft and hard ferromagnetic behavior by changing the parameter $\alpha=\alpha_{0}$. In order to explore how $\alpha_{0}$ affects the hysteresis loop in presence of interfacial effects, we have simulated magnetization curves considering different values of $\alpha_{0}$ for $z_{0} / L=0.5$, $l / L=0.5$, and $K=10$. Clearly, $\Delta M / M_{s}$ is reduced with the lowering of $\alpha_{0}$ (Fig. 3(a)), signifying that the change in the magnetic hysteresis due to interfacial effects would be lower in a hard ferromagnet. The impact of the pinning energy scale on the interfacial effect $\left(\Delta M / M_{s}\right)$ has been examined by varying the parameter $K$. The outcomes of our simulations with lowering $K$ have been shown in Fig. $3\left(\right.$ b) $\left[z_{0} / L=0.5, l / L=0.1\right.$, and $\left.\alpha_{0}=100\right]$. The hysteresis in $\Delta M / M_{s}$, which is very strong for the case of $K=20$, decreases with lowering $K$ and is completely absent for the $K=1$ case. Similar behaviors have been observed in $\rho_{\mathrm{THE}}$ vs. $H$ measurements at different $T$ for $\mathrm{SrRuO}_{3}[34,59]$.

\subsection{Effect of interfaces on both sides of the ferromagnet}

So far, we have investigated hysteresis of a ferromagnet with interfacial effects on one side while the other side retains its bulk-like interdomain magnetic coupling parameter. The inversion symmetry is broken in such a heterostructure, which can lead to finite D-M interaction. Since the net D-M interaction would vanish in case of a ferromagnet having interface on both sides, the possibilities of getting THE-like features has been also examined in $\mathrm{SrRuO}_{3}$-based heterostructures with such symmetric interfaces $[58,60]$. To explore the modification of hysteresis loop in such an artificial structure (see middle panel of Fig. 4(a)), we have considered the variation of $\alpha$ as a Gaussian function, where the center of the ferromagnetic compound is taken as the $z=0$ reference point. The variations of $\alpha=\alpha_{0} e^{-\left(z-z_{0}\right)^{2} / l^{\prime}}$ for different values of $l^{\prime}$ and $\alpha_{0}=100$ have been shown in left panel of Fig. 4(a). The corresponding variations of $\Delta M / M_{s}$ are shown in Fig. 4(b). Changes in magnetization due to interfacial effects are enhanced with the lowering of the parameter $l^{\prime}$. The overall behavior of $\Delta M / M_{s}$ vs. $H$ is very similar to the previously discussed ferromagnet having interfacial changes on one side. We also consider a possible scenario where $\alpha$ is enhanced towards the interface $\left[\alpha=\alpha_{0}+\alpha_{0}\left(1-e^{-\left(z-z_{0}\right)^{2} / l^{\prime}}\right)\right]$. The sign of $\Delta M / M_{s}$ is reversed compared to the previous case (Fig. 4(c)). Thus, our model predicts that THE-like features can be observed in a heterostructure with symmetric interfaces as well due to the layer dependent interdomain magnetic coupling even though the net D-M interaction is zero.

We further note that the observed maximum magnitude of topological Hall resistivity in experiment is around one order of magnitude smaller compared to the maximum magnitude of anomalous Hall resistivity $[37,58]$. Although we have not explicitly calculated resistivity here, the relative magnitudes of the extra AHE $(\propto \Delta M)$ and the AHE $(\propto M)$ component in present case can be used for the comparison. With the ranges of parameters we have explored in this paper, we have found that the maximum of $\Delta M / M_{s}$ is around one order of magnitude smaller compared to the maximum of $M / M_{s}$ as well.

\section{Conclusion}

To summarize, motivated by several recent experimental papers on $\mathrm{SrRuO}_{3}$ based heterostructures that claim that additional features in AHE that are often attributed to THE may not necessarily be a result of non trivial spin textures and may arise from an inhomogeneity in the ferromagnetic structure [40-44], we have developed a simple model to investigate changes in ferromagnetic hysteresis loop due to interfacial effects. Within the formalism of a phenomenological approach, developed by Jiles et al. [49], we have considered that the strength of the interdomain magnetic coupling parameter within the ferromagnetic material as a function of the distance from the interface. The resultant change in magnetization, obtained from our simulations, would lead to an additional contribution in AHE, which resembles experimental aspects of THE seen in several cases. While we do not claim that all experimental reports of THE in oxide heterostructures can be accounted 
(a)

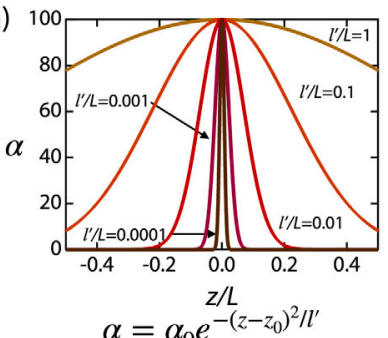

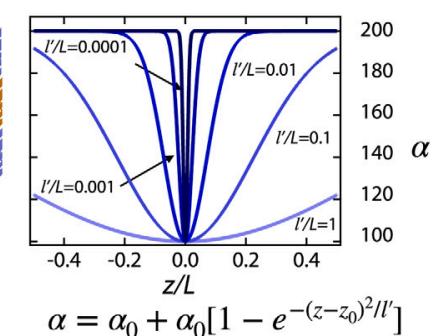

$\alpha=\alpha_{0}+\alpha_{0}\left[1-e^{-\left(z-z_{0}\right)^{2} / l^{\prime}}\right]$

(b)
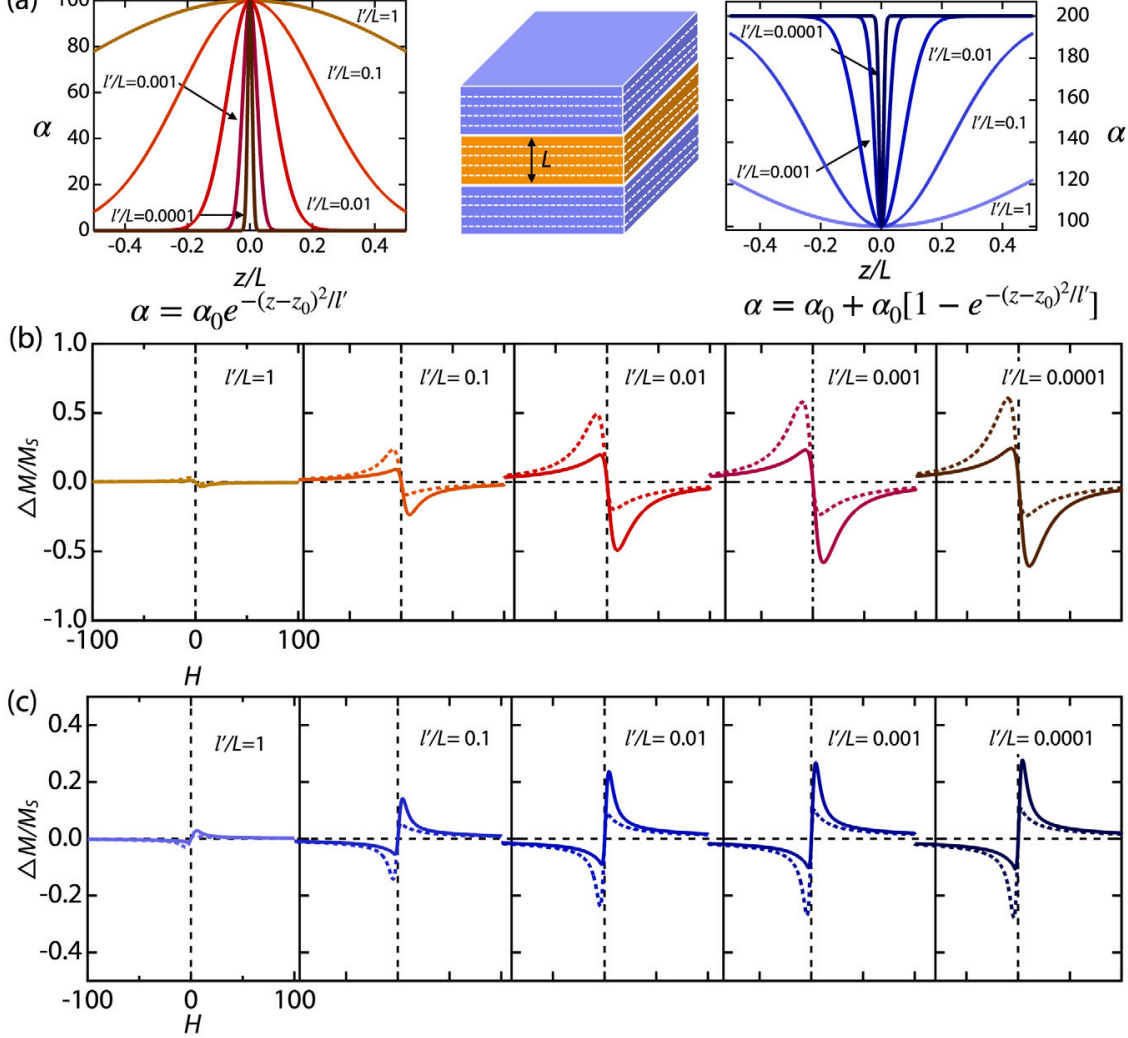

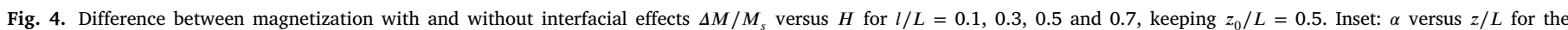
corresponding values of $l$. The dotted and solid line in (b) and (c) represents field sweep from $+H$ to $-H$, and $-H$ to $+H$, respectively.

by multi-channel AHE due to layer dependent hysteresis, we want to emphasize that such possibility must be examined before assigning these additional features in AHE to non trivial spin textures. Though we have discussed about interfacial effects in oxide heterostructure only in this paper, our present model is very general and can be applied for any heterostructure consisting of elemental ferromagnets, chalcogenides, heavy fermions etc.

See the supplementary material for simulations with larger range of $H$, and the effect of the parameter $l / L$ in the case of simulations having interfacial effects on one side.

\section{CRediT authorship contribution statement}

Nandana Bhattacharya: Coding and simulations, Data analysis, Writing - original draft. S. Middey: Conceptualization, Methodology, Data analysis, Writing - original draft.

\section{Declaration of competing interest}

The authors declare that they have no known competing financial interests or personal relationships that could have appeared to influence the work reported in this paper.

\section{Acknowledgments}

We thank Shashank Kumar Ojha and Prithwijit Mandal for insightful comments about this paper. SM acknowledges financial supports from DST Nanomission, India grant (Grant No. DST/NM/NS/2018/246) and Infosys Foundation, Bangalore.

\section{Appendix A. Supplementary data}

Supplementary material related to this article can be found online at https://doi.org/10.1016/j.jmmm.2021.168981.

\section{References}

[1] M. Imada, A. Fujimori, Y. Tokura, Metal-insulator transitions, Rev. Modern Phys. 70 (1998) 1039-1263, http://dx.doi.org/10.1103/RevModPhys.70.1039.

[2] Y. Tokura, Critical features of colossal magnetoresistive manganites, Rep. Progr. Phys. 69 (3) (2006) 797-851, http://dx.doi.org/10.1088/0034-4885/69/3/r06.

[3] B. Keimer, S.A. Kivelson, M.R. Norman, S. Uchida, J. Zaanen, From quantum matter to high-temperature superconductivity in copper oxides, Nature 518 (7538) (2015) 179-186, http://dx.doi.org/10.1038/nature14165.

[4] N.P. Armitage, P. Fournier, R.L. Greene, Progress and perspectives on electrondoped cuprates, Rev. Modern Phys. 82 (2010) 2421-2487, http://dx.doi.org/10. 1103/RevModPhys.82.2421.

[5] D.G. Schlom, L.-Q. Chen, X. Pan, A. Schmehl, M.A. Zurbuchen, A thin film approach to engineering functionality into oxides, J. Am. Ceram. Soc. 91 (8) (2008) 2429-2454, http://dx.doi.org/10.1111/j.1551-2916.2008.02556.x.

[6] P. Zubko, S. Gariglio, M. Gabay, P. Ghosez, J.-M. Triscone, Interface physics in complex oxide heterostructures, Ann. Rev. Condens. Matter Phys. 2 (2011) $141-165$.

[7] H.Y. Hwang, Y. Iwasa, M. Kawasaki, B. Keimer, N. Nagaosa, Y. Tokura, Emergent phenomena at oxide interfaces, Nature Mater. 11 (2012) 103-113, http://dx.doi. org/10.1038/nmat3223.

[8] J. Chakhalian, J.W. Freeland, A.J. Millis, C. Panagopoulos, J.M. Rondinelli, Colloquium: Emergent properties in plane view: Strong correlations at oxide interfaces, Rev. Modern Phys. 86 (2014) 1189-1202, http://dx.doi.org/10.1103/ RevModPhys.86.1189.

[9] A. Bhattacharya, S.J. May, Magnetic oxide heterostructures, Annu. Rev. Mater. Res. 44 (1) (2014) 65-90, http://dx.doi.org/10.1146/annurev-matsci-070813113447.

[10] S. Stemmer, S. James Allen, Two-dimensional electron gases at complex oxide interfaces, Annu. Rev. Mater. Res. 44 (1) (2014) 151-171, http://dx.doi.org/10. 1146/annurev-matsci-070813-113552.

[11] S. Middey, J. Chakhalian, P. Mahadevan, J.W. Freeland, A.J. Millis, D.D. Sarma, Physics of ultrathin films and heterostructures of rare-earth nickelates, Annu. Rev. Mater. Res. 46 (1) (2016) 305-334, http://dx.doi.org/10.1146/annurevmatsci-070115-032057.

[12] F. Hellman, A. Hoffmann, Y. Tserkovnyak, G.S.D. Beach, E.E. Fullerton, C Leighton, A.H. MacDonald, D.C. Ralph, D.A. Arena, H.A. Dür, P. Fischer, J. Grollier, J.P. Heremans, T. Jungwirth, A.V. Kimel, B. Koopmans, I.N. Krivorotov, S.J. May, A.K. Petford-Long, J.M. Rondinelli, N. Samarth, I.K. Schuller, A.N. Slavin, M.D. Stiles, O. Tchernyshyov, A. Thiaville, B.L. Zink, Interface-induced 
phenomena in magnetism, Rev. Modern Phys. 89 (2017) 025006, http://dx.doi. org/10.1103/RevModPhys.89.025006.

[13] J. Chakhalian, J.W. Freeland, G. Srajer, J. Strempfer, G. Khaliullin, J.C. Cezar, T. Charlton, R. Dalgliesh, C. Bernhard, G. Cristiani, H.U. Habermeier, B. Keimer, Magnetism at the interface between ferromagnetic and superconducting oxides, Nat Phys. 2 (4) (2006) 244-248, http://dx.doi.org/10.1038/nphys272.

[14] J. Chakhalian, J.W. Freeland, H.-U. Habermeier, G. Cristiani, G. Khaliullin, M. van Veenendaal, B. Keimer, Orbital reconstruction and covalent bonding at an oxide interface, Science 318 (5853) (2007) 1114-1117, http://dx.doi.org/ 10.1126/science.1149338, http://www.sciencemag.org/content/318/5853/1114. abstract.

[15] A. Brinkman, M. Huijben, M. van Zalk, J. Huijben, U. Zeitler, J.C. Maan, W.G. van der Wiel, G. Rijnders, D.H.A. Blank, H. Hilgenkamp, Magnetic effects at the interface between non-magnetic oxides, Nature Mater. 6 (7) (2007) 493-496, http://dx.doi.org/10.1038/nmat1931.

[16] S.J. May, P.J. Ryan, J.L. Robertson, J.-W. Kim, T.S. Santos, E. Karapetrova, J.L. Zarestky, X. Zhai, S.G.E. te Velthuis, J.N. Eckstein, S.D. Bader, A. Bhattacharya, Enhanced ordering temperatures in antiferromagnetic manganite superlattices, Nature Mater. 8 (11) (2009) 892-897, http://dx.doi.org/10.1038/nmat2557.

[17] A.V. Boris, Y. Matiks, E. Benckiser, A. Frano, P. Popovich, V. Hinkov, P. Wochner, M. Castro-Colin, E. Detemple, V.K. Malik, C. Bernhard, T. Prokscha, A. Suter, Z. Salman, E. Morenzoni, G. Cristiani, H.-U. Habermeier, B. Keimer, Dimensionality control of electronic phase transitions in nickel-oxide superlattices, Science 332 (6032) (2011) 937-940, http://dx.doi.org/10.1126/science.1202647, http: //www.sciencemag.org/content/332/6032/937.abstract.

[18] C. He, A.J. Grutter, M. Gu, N.D. Browning, Y. Takamura, B.J. Kirby, J.A. Borchers, J.W. Kim, M.R. Fitzsimmons, X. Zhai, V.V. Mehta, F.J. Wong, Y. Suzuki, Interfacia ferromagnetism and exchange bias in $\mathrm{caruo}_{3} / \mathrm{camno}_{3}$ superlattices, Phys. Rev. Lett. 109 (2012) 197202, http://dx.doi.org/10.1103/PhysRevLett.109. 197202.

[19] M. Gibert, P. Zubko, R. Scherwitzl, J. Íñguez, J.-M. Triscone, Exchange bias in $\mathrm{LaNiO}_{3}-\mathrm{LaMnO}_{3}$ superlattices, Nat. Mater. 11 (3) (2012) 195-198, http://dx.doi. org/10.1038/nmat3224.

[20] A.J. Grutter, H. Yang, B.J. Kirby, M.R. Fitzsimmons, J.A. Aguiar, N.D. Browning, C.A. Jenkins, E. Arenholz, V.V. Mehta, U.S. Alaan, Y. Suzuki, Interfacial ferromagnetism in $\mathrm{LaNiO}_{3} / \mathrm{CaMnO}_{3}$ superlattices, Phys. Rev. Lett. 111 (2013) 087202, http://dx.doi.org/10.1103/PhysRevLett.111.087202.

[21] E.J. Moon, R. Colby, Q. Wang, E. Karapetrova, C.M. Schlepütz, M.R. Fitzsimmons, S.J. May, Spatial control of functional properties via octahedral modulations in complex oxide superlattices, Nature Commun. 5 (1) (2014) http://dx.doi.org/10. 1038/ncomms6710.

[22] J.D. Hoffman, B.J. Kirby, J. Kwon, G. Fabbris, D. Meyers, J.W. Freeland, I. Martin, O.G. Heinonen, P. Steadman, H. Zhou, C.M. Schlepütz, M.P.M. Dean, S.G.E. te Velthuis, J.-M. Zuo, A. Bhattacharya, Oscillatory noncollinear magnetism induced by interfacial charge transfer in superlattices composed of metallic oxides, Phys. Rev. X 6 (2016) 041038, http://dx.doi.org/10.1103/ PhysRevX.6.041038.

[23] R.K. Patel, D. Meyers, X. Liu, P. Mandal, M. Kareev, P. Shafer, J.-W. Kim, P.J. Ryan, S. Middey, J. Chakhalian, Emergent behavior of lanio3 in short-periodic nickelate superlattices, APL Mater. 8 (4) (2020) 041113, http://dx.doi.org/10. 1063/5.0004530.

[24] D. Yi, J. Liu, S.-L. Hsu, L. Zhang, Y. Choi, J.-W. Kim, Z. Chen, J.D. Clarkson, C.R. Serrao, E. Arenholz, P.J. Ryan, H. Xu, R.J. Birgeneau, R. Ramesh, Atomicscale control of magnetic anisotropy via novel spin-orbit coupling effect in la2/3sr1/3mno3/SrIrO3 superlattices, Proc. Natl. Acad. Sci. 113 (23) (2016) 6397-6402, http://dx.doi.org/10.1073/pnas.1524689113.

[25] J. Matsuno, N. Ogawa, K. Yasuda, F. Kagawa, W. Koshibae, N. Nagaosa, Y. Tokura, M. Kawasaki, Interface-driven topological hall effect in SrRuO3-SrIrO3 bilayer, Sci. Adv. 2 (7) (2016) e1600304, http://dx.doi.org/10.1126/sciadv. 1600304.

[26] A. Neubauer, C. Pfleiderer, B. Binz, A. Rosch, R. Ritz, P.G. Niklowitz, P. Böni, Topological hall effect in the $a$ phase of mnsi, Phys. Rev. Lett. 102 (2009) 186602, http://dx.doi.org/10.1103/PhysRevLett.102.186602.

[27] N. Kanazawa, Y. Onose, T. Arima, D. Okuyama, K. Ohoyama, S. Wakimoto, K. Kakurai, S. Ishiwata, Y. Tokura, Large topological hall effect in a short-period helimagnet mnge, Phys. Rev. Lett. 106 (2011) 156603, http://dx.doi.org/10. 1103/PhysRevLett.106.156603.

[28] C. Sürgers, G. Fischer, P. Winkel, H. v. Löhneysen, Large topological hall effect in the non-collinear phase of an antiferromagnet, Nature Commun. 5 (1) (2014) http://dx.doi.org/10.1038/ncomms4400.

[29] T. Kurumaji, T. Nakajima, M. Hirschberger, A. Kikkawa, Y. Yamasaki, H. Sagayama, H. Nakao, Y. Taguchi, T. hisa Arima, Y. Tokura, Skyrmion lattice with a giant topological hall effect in a frustrated triangular-lattice magnet, Science 365 (6456) (2019) 914-918, http://dx.doi.org/10.1126/science.aau0968.

[30] C. Liu, Y. Zang, W. Ruan, Y. Gong, K. He, X. Ma, Q.-K. Xue, Y. Wang, Dimensional crossover-induced topological hall effect in a magnetic topological insulator, Phys. Rev. Lett. 119 (2017) 176809, http://dx.doi.org/10.1103/PhysRevLett.119. 176809.
[31] S.K. Ojha, S.K. Gogoi, M.M. Patidar, R.K. Patel, P. Mandal, S. Kumar, R. Venkatesh, V. Ganesan, M. Jain, S. Middey, Oxygen vacancy-induced topological hall effect in a nonmagnetic band insulator, Adv. Quantum Technol. 3 (7) (2020) 2000021, http://dx.doi.org/10.1002/qute.202000021.

[32] L. Vistoli, W. Wang, A. Sander, Q. Zhu, B. Casals, R. Cichelero, A. Barthélémy, S. Fusil, G. Herranz, S. Valencia, R. Abrudan, E. Weschke, K. Nakazawa, H. Kohno, J. Santamaria, W. Wu, V. Garcia, M. Bibes, Giant topological hall effect in correlated oxide thin films, Nat. Phys. 15 (1) (2018) 67-72, http: //dx.doi.org/10.1038/s41567-018-0307-5.

[33] Q. Shao, Y. Liu, G. Yu, S.K. Kim, X. Che, C. Tang, Q.L. He, Y. Tserkovnyak, J. Shi, K.L. Wang, Topological hall effect at above room temperature in heterostructures composed of a magnetic insulator and a heavy metal, Nat. Electron. 2 (5) (2019) 182-186, http://dx.doi.org/10.1038/s41928-019-0246-x.

[34] E. Skoropata, J. Nichols, J.M. Ok, R.V. Chopdekar, E.S. Choi, A. Rastogi, C. Sohn, X. Gao, S. Yoon, T. Farmer, R.D. Desautels, Y. Choi, D. Haskel, J.W. Freeland, S. Okamoto, M. Brahlek, H.N. Lee, Interfacial tuning of chiral magnetic interactions for large topological hall effects in LaMnO3/SrIrO3 heterostructures, Sci. Adv. 6 (27) (2020) eaaz3902, http://dx.doi.org/10.1126/sciadv.aaz3902.

[35] G. Cao, S. McCall, M. Shepard, J.E. Crow, R.P. Guertin, Thermal, magnetic, and transport properties of single-crystal $\mathrm{sr}_{1-x} \mathrm{Ca}_{x} \mathrm{ruo}_{3}(0<\mathrm{x}<1.0)$, Phys. Rev. B 56 (1997) 321-329, http://dx.doi.org/10.1103/PhysRevB.56.321.

[36] Y. Ohuchi, J. Matsuno, N. Ogawa, Y. Kozuka, M. Uchida, Y. Tokura, M. Kawasaki, Electric-field control of anomalous and topological hall effects in oxide bilayer thin films, Nature Commun. 9 (1) (2018) http://dx.doi.org/10.1038/s41467-01702629-3.

[37] L. Wang, Q. Feng, Y. Kim, R. Kim, K.H. Lee, S.D. Pollard, Y.J. Shin, H. Zhou, W. Peng, D. Lee, W. Meng, H. Yang, J.H. Han, M. Kim, Q. Lu, T.W. Noh, Ferroelectrically tunable magnetic skyrmions in ultrathin oxide heterostructures, Nature Mater. 17 (12) (2018) 1087-1094, http://dx.doi.org/10.1038/s41563018-0204-4.

[38] Q. Qin, L. Liu, W. Lin, X. Shu, Q. Xie, Z. Lim, C. Li, S. He, G.M. Chow, J. Chen, Emergence of topological hall effect in a SrRuO3 single layer, Adv. Mater. 31 (8) (2019) 1807008, http://dx.doi.org/10.1002/adma.201807008.

[39] K.-Y. Meng, A.S. Ahmed, M. Baćani, A.-O. Mandru, X. Zhao, N. Bagués, B.D. Esser, J. Flores, D.W. McComb, H.J. Hug, F. Yang, Observation of nanoscale skyrmions in SrIrO3/SrRuO3 bilayers, Nano Lett. 19 (5) (2019) 3169-3175, http://dx.doi.org/10.1021/acs.nanolett.9b00596.

[40] D. Kan, T. Moriyama, K. Kobayashi, Y. Shimakawa, Alternative to the topological interpretation of the transverse resistivity anomalies in srruo $_{3}$, Phys. Rev. B 98 (2018) 180408, http://dx.doi.org/10.1103/PhysRevB.98.180408.

[41] L. Wu, F. Wen, Y. Fu, J.H. Wilson, X. Liu, Y. Zhang, D.M. Vasiukov, M.S. Kareev, J.H. Pixley, J. Chakhalian, Berry phase manipulation in ultrathin SrRuO3 films, Phys. Rev. B 102 (22) (2020) 220406, http://dx.doi.org/10.1103/physrevb.102. 220406.

[42] G. Kimbell, P.M. Sass, B. Woltjes, E.K. Ko, T.W. Noh, W. Wu, J.W.A. Robinson, Two-channel anomalous hall effect in srruo $_{3}$, Phys. Rev. Mater. 4 (2020) 054414, http://dx.doi.org/10.1103/PhysRevMaterials.4.054414.

[43] L. Wang, Q. Feng, H.G. Lee, E.K. Ko, Q. Lu, T.W. Noh, Controllable thickness inhomogeneity and berry curvature engineering of anomalous hall effect in srruo3 ultrathin films, Nano Lett. 20 (4) (2020) 2468-2477, http://dx.doi.org/ 10.1021/acs.nanolett.9b05206.

[44] L. Wysocki, L. Yang, F. Gunkel, R. Dittmann, P.H.M. van Loosdrecht, I. LindforsVrejoiu, Validity of magnetotransport detection of skyrmions in epitaxial $\mathrm{srruo}_{3}$ heterostructures, Phys. Rev. Mater. 4 (2020) 054402, http://dx.doi.org/10.1103/ PhysRevMaterials.4.054402.

[45] D.J. Groenendijk, C. Autieri, T.C. van Thiel, W. Brzezicki, J. Hortensius, D. Afanasiev, N. Gauquelin, P. Barone, K. van den Bos, S. van Aert, et al., Berry phase engineering at oxide interfaces, Phys. Rev. Res. (2020) 023404.

[46] A. Alberca, N.M. Nemes, F.J. Mompean, N. Biskup, A. de Andres, C. Munuera, J. Tornos, C. Leon, A. Hernando, P. Ferrer, G.R. Castro, J. Santamaria, M. GarciaHernandez, Exotic magnetic anisotropy map in epitaxial $\mathrm{la}_{0.7} \mathrm{ca}_{0.3} \mathrm{mno}_{3}$ films on batio $_{3}$, Phys. Rev. B 84 (2011) 134402, http://dx.doi.org/10.1103/PhysRevB.84. 134402.

[47] F.Y. Bruno, J. Garcia-Barriocanal, M. Varela, N.M. Nemes, P. Thakur, J.C. Cezar, N.B. Brookes, A. Rivera-Calzada, M. Garcia-Hernandez, C. Leon, S. Okamoto, S.J. Pennycook, J. Santamaria, Electronic and magnetic reconstructions in $\mathrm{la}_{0.7} \mathrm{sr}_{0.3} \mathrm{mno}_{3} / \mathrm{srtio}_{3}$ heterostructures: A case of enhanced interlayer coupling controlled by the interface, Phys. Rev. Lett. 106 (2011) 147205, http://dx.doi. org/10.1103/PhysRevLett.106.147205.

[48] S. Singh, M.R. Fitzsimmons, T. Lookman, J.D. Thompson, H. Jeen, A. Biswas, M.A. Roldan, M. Varela, Magnetic nonuniformity and thermal hysteresis of magnetism in a manganite thin film, Phys. Rev. Lett. 108 (2012) 077207, http://dx.doi.org/10.1103/PhysRevLett.108.077207.

[49] D. Jiles, D. Atherton, Theory of ferromagnetic hysteresis, J. Magn. Magn. Mater. 61 (1-2) (1986) 48-60, http://dx.doi.org/10.1016/0304-8853(86)90066-1.

[50] Z. Liu, A. Burigu, Y. Zhang, H.M. Jafri, X. Ma, E. Liu, W. Wang, G. Wu, Giant topological hall effect in tetragonal heusler alloy mn2ptsn, Scr. Mater. 143 (2018) 122-125, http://dx.doi.org/10.1016/j.scriptamat.2017.09.024. 
[51] W. Wang, Y.-F. Zhao, F. Wang, M.W. Daniels, C.-Z. Chang, J. Zang, D. Xiao, W. $\mathrm{Wu}$, Chiral-bubble-induced topological hall effect in ferromagnetic topological insulator heterostructures, Nano Lett. 21 (2) (2021) 1108-1114, http://dx.doi. org/10.1021/acs.nanolett.0c04567.

[52] Y. Li, L. Zhang, Q. Zhang, C. Li, T. Yang, Y. Deng, L. Gu, D. Wu, Emergent topological hall effect in la0. 7sr0. 3mno3/sriro3 heterostructures, ACS Appl. Mater. Interfaces 11 (23) (2019) 21268-21274, http://dx.doi.org/10.1021/ acsami.9b05562.

[53] Y. Yun, Y. Ma, T. Su, W. Xing, Y. Chen, Y. Yao, R. Cai, W. Yuan, W. Han, Role of la doping for topological hall effect in epitaxial euo films, Phys. Rev. Mater. 2 (2018) 034201, http://dx.doi.org/10.1103/PhysRevMaterials.2.034201.

[54] P. Langevin, Magnétisme et théorie des électrons, Ann. Chim. Et Phys. (8) (1905) 203.

[55] Z. Li, Z. Qiao, T. Tang, Numerical Solution of Differential Equations: Introduction To Finite Difference and Finite Element Methods, Cambridge University Press, 2017.
[56] A. Ohtomo, D.A. Muller, J.L. Grazul, H.Y. Hwang, Artificial charge-modulationin atomic-scale perovskite titanate superlattices, Nature 419 (6905) (2002) 378-380, http://dx.doi.org/10.1038/nature00977.

[57] Y. Liu, X. Ke, Cheminform abstract: Interfacial magnetism in complex oxide heterostructures probed by neutrons and x-rays, ChemInform 46 (47) (2015) 373003, http://dx.doi.org/10.1002/chin.201547213.

[58] W. Wang, M.W. Daniels, Z. Liao, Y. Zhao, J. Wang, G. Koster, G. Rijnders, C.-Z. Chang, D. Xiao, W. Wu, Spin chirality fluctuation in two-dimensional ferromagnets with perpendicular magnetic anisotropy, Nature Mater. 18 (10) (2019) 1054-1059, http://dx.doi.org/10.1038/s41563-019-0454-9.

[59] S.D. Seddon, D.E. Dogaru, S.J.R. Holt, D. Rusu, J.J.P. Peters, A.M. Sanchez, M. Alexe, Real-space observation of ferroelectrically induced magnetic spin crystal in SrRuO3, Nature Commun. 12 (1) (2021) http://dx.doi.org/10.1038/s41467021-22165-5.

[60] L. Yang, L. Wysocki, J. Schöpf, L. Jin, A. Kovács, F. Gunkel, R. Dittmann, P.H.M. van Loosdrecht, I. Lindfors-Vrejoiu, Origin of the hump anomalies in the hall resistance loops of ultrathin $\mathrm{srruo}_{3} / \mathrm{sriro}_{3}$ multilayers, Phys. Rev. Mater. 5 (2021) 014403, http://dx.doi.org/10.1103/PhysRevMaterials.5.014403. 